\title{
étude épidémiologique de la toxoplasmose chez les femmes enceintes à Amiens (Picardie) nécessité d'une enquête nationale
}

\author{
B Carme 1, E Lenne 1, V Tirard 1,2, M P Hayette 1, J Gondry ${ }^{3}$ \\ 1 Service de parasitologie-mycologie, CHU d'Amiens, 80054 Amiens ; ${ }^{2}$ Direction générale de la santé ; \\ ${ }^{3}$ Senvice de gynécologie obstétrique, $\mathrm{CHU}$ d'Amiens
}

\begin{abstract}
introduction
La toxoplasmose congénitale bénéficie en France d'un programme important de prévention primaire mais, paradoxalement aucune évaluation objective à large échelle de ce programme n'a encore été réalisée, De plus, les notions communément admises sur l'épidémiologie de la toxoplasmose chez les femmes enceintes reposent sur des données relativement anciennes [1]. En outre, le caractère hétérogène des protocoles utilisés et des populations enquêtées rend difficile la comparaison des résultats. Une évaluation de la situation actuelle à un niveau national ainsi qu'un effort de standardisation sont souhaitables.

L'enquête, décrite dans cet article, réalisée à Amiens avait pour objectifs l'évaluation d'un niveau d'immunité vis-àvis de la toxoplasmose des femmes venant d'accoucher confronté aux facteurs d'exposition et celle de l'état des connaissances des mesures préventives pour les femmes non immunes et de leur mise en pratique.
\end{abstract}

\section{méthodologie}

Il s'agit d'une enquête prospective menée à la maternité du CHU d'Amiens du $1^{\text {er }}$ mars 1993 au 28 février 1994 auprès des femmes venant d'accoucher (grossesse > à 28 semaines d'aménorrhée). Une femme sur deux a été sélectionnée selon les numéros d'enregistrement des accouchements (numéros pairs retenus).
Le dossier médical a été consulté avec le relevé de la sérologie toxoplasmique (seuil d'immunité retenu $10 \mathrm{UI} / \mathrm{ml}$ quelle que soit la technique sérologique mise en ouvre) et un contrôle sérologique a eu lieu en cas d'absence de données. L'interrogatoire de la patiente en post-partum a toujours été réalisé par le même enquêteur selon un questionnaire de 40 items destiné à obtenir des informations d'ordre général et des données spécifiques à la toxoplasmose. Certaines questions se rapportant aux mesures préventives n'ont été posées que chez les femmes non immunes. L'analyse des données s'est faite sur le logiciel Epidémio B Duflo, version 1988.

\section{résultats}

\section{population enquêtée}

987 femmes ont été enquêtées. Les répartitions par âge et par catégorie socioprofessionnelle sont conformes à celles de l'ensemble des femmes en issue de grossesse du département de la Somme selon les données de l'INSEE disponibles pour l'année 1993. Pour les 886 femmes françaises, la séroprévalence visà-vis de la toxoplasmose est analysée en fonction de différents facteurs : cuisson de la viande, présence d'un chat à domicile... Ceci n'a pas été possible pour les 101 femmes étrangères, pour lesquelles seul le chiffre de séroprévalence est donné, en raison des faibles effectifs par sous-groupe.

\section{séroprévalence}

La séroprévalence des 886 femmes françaises est globalement de $58 \%$ (intervalle de confiance [IC] : 55-61). Elle augmente avec l'âge, passant de $35 \%$ pour les femmes de moins de 20 ans à $74 \%$ pour celles de plus de 35 ans (tableau I). Pour les 101 femmes étrangères la séroprévalence vis-à-vis de la toxoplasmose est de $61 \%$ (intervalle de conflance : $51-70$ )

\begin{tabular}{|c|c|c|c|c|}
\hline Age & Sujets: & & Séropositivité & \\
\hline & & Nombre & $\%$ & $I C$ \\
\hline $\begin{array}{l}<20 \\
20-24 \\
25-29 \\
30-34 \\
>35\end{array}$ & $\begin{array}{r}43 \\
193 \\
339 \\
216 \\
95\end{array}$ & $\begin{array}{r}15 \\
93 \\
195 \\
137 \\
30\end{array}$ & $\begin{array}{l}35 \\
48 \\
57 \\
63 \\
74\end{array}$ & $\begin{array}{l}21-49 \\
41-55 \\
52-62 \\
57-69 \\
65-83\end{array}$ \\
\hline
\end{tabular}




\section{immunité et facteurs d'exposition}

Dans notre enquête, parmi les facteurs d'exposition au toxoplasme, seules la consommation de viande pas toujours bien cuite (odds ratio $=2.7, \mathrm{IC} 2,0-3,6$ ) ainsi que la présence d'un chat dans l'entourage immćdiat, dans le passé ou actuellement (odds ratio $=1.4, \mathrm{IC} 1,0$ 2,0 ) sont associées à la séropositivité visà-vis de la toxoplasmose. L'analyse par sous-groupe (OR de Mantel-Haenszel) (tableau II) montre que ces deux facteurs agissent indépendamment l'un de l'autre sur le statut immunitaire vis-à-vis de la toxoplasmose. Par contrc, on ne retrouve aucune différence significative pour les autres facteurs analysés : type d'habitat (urbain, rural), niveau socioculturel (niveau d'études et profession), habitude de conserver la viande congelée, prise habituelle de repas à l'extéricur... Soulignons que ces données se rapportent au statut immunitaire ct non aux séroconservations en cours de grossesse.

\section{contaminations récentes probables et séroconversions}

Les quatre cas de contamination retenus dans cette enquête, sur $376 \mathrm{fcmmes}$ non immunes $(1,1 \%)$, sont tous survenus en période périconceptionnelle ou au cours du premier trimestre.

\section{connaissances et observance des mesures préventives}

Dans cette enquête, la quasi-totalité des femmes non immunes a des notions sur la toxoplasmose (96\%), sait qu'il s'agit d'un danger potentiel (96\%) et peut citer spontanément au moins deux mesures préventives contre l'infection; les recommandations classiques scraient largement suivies (tableau III).

A noter quc la proportion de femmes suivies à la maternité du C.HU est de $60 \%$; chacune d'entre elles ayant reçu une information dès sa première visite avec la remisc d'une fiche, et que l'absence de sérologie toxoplasmique n'a ćté objectivée que dans $1 \%$ des cas. Il s'agissait de grossesses non suivies, non déclarécs.

Tableau H. Seroprevalence selon les deux pringlpatax focteurs d'expostion.

\begin{tabular}{lccccc} 
Facteurs & Total $(n)$ & \multicolumn{5}{c}{ Séropositivité } \\
& & Nombre & $\%$ & OR & $I C$ \\
Chat : & & & & & \\
- char + & 212 & 136 & 64 & $1,4^{* *}$ & $1,0-2 ; 0$ \\
- chat - & 671 & 373 & 56 & & \\
Cuissen viande : & & & & & \\
- cuisson + & 337 & 144 & 43 & $2,7^{*}$ & $2,0-3,6$ \\
- cuisson - & 546 & 365 & 67 & &
\end{tabular}

* OR ajusté (Mantel Haenszel) pour le facteur cuisson : $1,7(1,2-2,3)$.

** OR ajusté (Mantel Haenszel) pour le factcur chat : 2,6 (2,0-3,5).

Chat + : possession d'un chat.

Chat - : pas de chat.

Cuisson + : consommation de viande toujours bien cuite.

Cuisson - : consommation de viande pas toujours bien cuite.

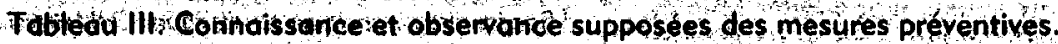

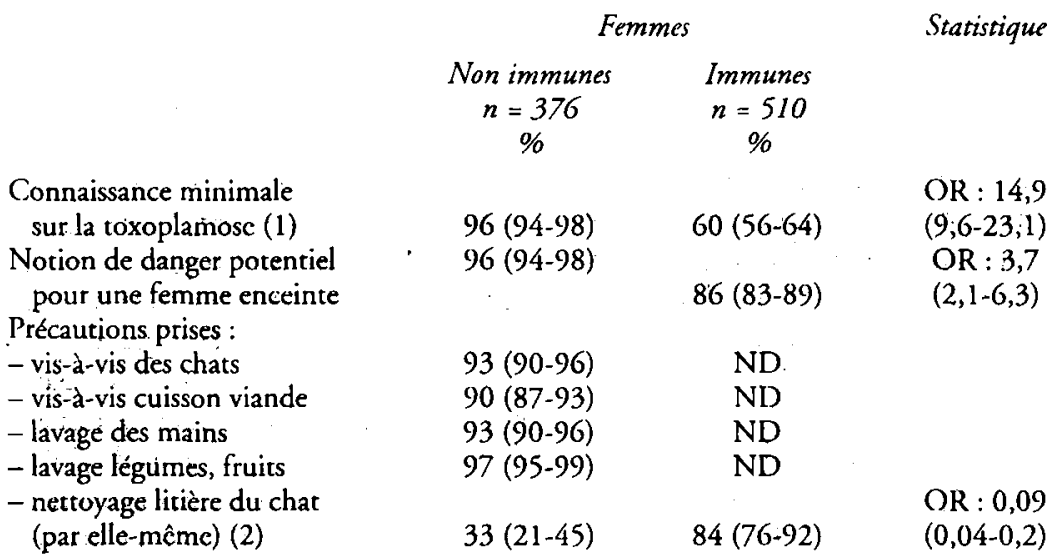

$\mathrm{OR}$ : odds ratio, ND : non disponible, les valeurs entre parenthèses correspondent à l'intervallc de confiance.

(1) Peuvent citer au moins deux modes de contamination en réponse à une question ouverte ;

(2) Pour les femmes ayant gardé leur chat.

\section{discussion}

Le bilan effectué en 1983 au niveau national montrait une grande variabilité de séroprévalence avec des taux de 20 à $66 \%$ selon les régions; la valeur moyennc ćtant de $50 \%$ [2]. Les enquêtes concernant le niveau d'immunité toxoplasmique sont souvent basćes sur les relevés sérologiques des laboratoires. $\mathrm{Or}$, il existe des biais de recrutement importants, dont le plus évident est quc les femmes dont l'immunité antérieure est connue n'ont plus à ĉtre prélevées et se trouvent de la sorte sous-représentées.

La notion classique d'une diminution de la séroprévalence en France depuis une trentainc d'année s'appuie essentiellement sur les données parisiennes fai- sant état d'une réduction de près de $30 \%$ entre 1960 et 1983 . Cette tendance n'cst pas retrouvée à Amiens puisqu'unc étude réaliséc en 1970 chez 291 donneurs de sang âgés de 20 à 40 ans objectivait une séroprévalence toxoplasmique de 64\% [3], ni à Toulouse [4], ni même à Paris seloq une étude parue en 1987 où le taux proposé est de $71 \%$ [5]. Toutefois, le vieillisscment de la population des fermmes enceintes, sensible depuis 20 ans, peut introduire un biais dans l'évaluation de cette tendance si les comparaisons se font sans ajustement par I'âge.

Les deux facteurs de risque habituels de la toxoplasmose (la cuisson de la viande et le contact avec un chat) sont retrouvés dans cette enquête. Cepen- 
dant, cette association peut être le fait d'un biais du fait de la nature transversale de l'enquête (les femmes qui se savent séropositives pour la toxoplasmose n'ont plus à prendre de précautions au cours de leur grossesse).

La proportion de femmes immunes augmente progressivement en fonction de l'âge ; ce qui témoigne de la survenue régulière d'infection toxoplasmique chez l'adulte. Ce raisonnement implique toutefois que l'exposition au parasite ne differe pas en fonction de l'âge, entre 20 et 40 ans, et de l'époque. Ce qui semble être le cas à Amiens où, selon les valeurs obtenues, l'incidence annuelle des séroconversions serait de l'ordre de $2 \%$ par an, soit de $0,5 \%$ par trimestre. Cette valeur est proche de celle retrouvée dans notre enquête au cours du premier trimestre de la grossesse, période où ont eu lieu les quatre cas de contamination très probable.

Le nombre d'observations de cas de séroconversion est trop faible pour pouvoir en tirer des conclusions. En effet, pour évaluer l'incidence des contaminations récentes, il faut un nombre d'observations suffisant. Une étude cas-témoin étant nécessaire pour en connaître les facteurs de risque. En dehors des cas de séroconversion dûment constatés (sérologie négative puis positive à un contrôle ultérieur), il est parfois difficile de conclure même en ayant accès au dossier médical [5]. En effet, les arguments sérologiques disponibles, en particulier en cas de contamination au cours du premier trimestre, ne sont pas toujours déterminants, faute de sérologie récente de référence.

Pendant les 12 mois de l'enquête une seule interruption thérapeutique de grossesse pour toxoplasmose a été pratiquée dans le service.

Au cours d'une précédente enquête réalisée à Amiens sur plus de 6000 femmes ayant accouché dans ce même service entre 1984 et 1987 , les taux se situaient entre 0,72 et $1,13 \%$ selon la plus ou moins grande rigueur observée pour affirmer la contamination au cours de la grossesse [6]. Les valeurs habituellement rapportées varient entre 5 et $20 \%$.

On pourrait être tenté d'attribuer l'absence de séroconversion au cours des deuxième et troisième trimestres à l'efficacité de la surveillance et des mesures préventives préconisées. Une étude plus large est nécessaire. Si cette notion se confirmait, la nécessité d'une information précoce afin d'éviter les séroconversions de la période périconceptionnelle et du premier trimestre n'en deviendrait que plus évidente.

En $1986,69 \%$ des 1671 femmes enquêtées à Paris connaissaient au moins deux mesures préventives et $88 \%$ des non immunes avaient connaissance du danger [7]. Nos résultats difficilement comparables apparaissent cependant plus favorables.

\section{conclusion}

Les résultats obtenus au cours de cette enquête ont permis d'actualiser et de préciser certains aspects épidémiologiques de la toxoplasmose à Amiens mais ne peuvent être généralisés à un niveau national.

Cette étude a été entreprise avec l'espoir d'intéresser les organismes chargés de l'évaluation des problèmes de Santé publique et de favoriser ainsi la mise en place d'une enquête plus large. Cela devrait se concrétiser en 1995 du fait de la programmation d'une étude nationale devant se dérouler au niveau des maternités et être coordonnée par la Direction générale de la Santé et le Réseau national de Santé publique.

Le choix d'un protocole impliquant le recueil des données auprès des femmes en issue de grossesse présente plusieurs avantages en facilitant :

- la standardisation des protocoles et donc la comparaison des résultats dans l'espace et dans le temps ;
- l'évaluation des cas de contamination en cours de grossesse, en évitant les doublons, par accès direct au dossier médical ;

- la réalisation d'études cas-témoin.

De plus, il permet d'intégrer les femmes non ou mal suivies sérologiquement. Par contre, l'exclusion des femmes n'ayant pas poursuivi leur grossesse jusqu'à son terme est un facteur de sousestimation des contaminations. Cellesci pourront être évaluées par des études complémentaires au sein des mêmes services hospitaliers.

Extrait du Bulletin épidémiologique hebdomadaire, $\mathrm{n}^{\circ} 38-1994$

\section{références}

1 Bougnoux ME, Hubert B. Toxoplasmose congénitale. Bilan de la prévention primaire en France Bull Epidemiol Hebdo 1990;4:13-4

2 LNS. Immunité et infection toxoplasmique de la femme enceinte en France, Bull Epidemiol Hebdo 1984:51

3 Poulain C. Diagnostic épidémiologique de la toxoplasmose dans le département de la Somme. Thèse médecine, Amiens 1970

4 Espeillac D, Malavaud $S$, Bessières $M H$, Grandjean $H$. Étude séroépidémiologique vis-à-vis de la toxoplasmose chez la femme enceinte dans la région toulousaine. Méd Mal Infect 1989; 19:80-2

5 Jeannel D, Niel G, Costagliola D et al. Epidemiology of toxoplasmosis among pregnant women in the Paris area. Int J Epidemiol 1988;17:595602

6 Gondry J, Chandenier J, Mida Met al. Problèmes pratiques posés par la toxoplasmose en cours de grossesse. Rev Fr Gynéc 1989;84:635-43

7 Goulet V, Le Magny F, Iborra M. Enquête sur la connaissance des mesures préventives contre la toxoplasmose auprès des femmes venant d'accoucher. Bull Epidemiol Hebdo. 1990; 4: 14-5 